\title{
EDUCAÇÃO CONTINUADA DA EQUIPE DE ENFERMAGEM NAS UTIS DO MUNICÍPIO DE SÃO PAULO*
}

\author{
Maria Sumie Koizumi** \\ Miako Kimura*** \\ Ana Maria Kazue Miyadahira*** \\ Diná de Almeida Lopes Monteiro da Cruz*** \\ Katia Grillo Padilha*** \\ Regina Marcia Cardoso de Sousa*** \\ Paula Dal Maso Altimari***
}

KOIZUMI, M.S.; KIMURA, M.; MIYADAHIRA, A.M.K.; CRUZ, D.de A.L.M.da; PADILHA, K.G.; SOUSA,

R.M.C.de; ALTIMARI, P.D.M. Educação continuada da equipe de enfermagem nas UTIs do município de São

Paulo. Rev.latino-am.enfermagem, Ribeirão Preto, v. 6, n. 3, p. 33-41, julho 1998.

Este estudo é parte de um projeto maior sobre os recursos estruturais das UTIs no Município de São Paulo, incluindo a análise de sua estrutura física, recursos humanos, materiais e equipamentos. Os aspectos relativos às atividades de educação continuada da equipe de enfermagem são analisados neste artigo. A amostra estudada foi de 43 UTIs e um questionário respondido pela enfermeira responsável pela Unidade, o instrumento usado para a coleta de dados. Do total de Unidades estudadas, $34(79,1 \%)$ responderam ter treinamento inicial especifico e 18 (41,9\%), programas de atualização, os quais enfocam principalmente, revisão de técnicas e rotinas e atualização de patologias. As atividades de educação continuada são desenvolvidas predominantemente por enfermeiros das UTIs. Em relação aos enfermeiros, além das atividades de educação continuada desenvolvidas pelo próprio hospital, 50,2\% assinalaram ter feito cursos de especialização ou aprimoramento em enfermagem médico-cirúrgica ou em outras áreas ou tanto em enfermagem médico-cirúrgica como em outras áreas. Face ao detectado e considerando o desenvolvimento do enfermeiro de UTI como o de um especialista, algumas sugestões para o aprimoramento das atividades de educação continuada nessas Unidades são apresentadas.

UNITERMOS: educação continuada, equipe de enfermagem, unidades de terapia intensiva

\section{INTRODUÇÃO}

As atividades de educação continuada efetivamente desenvolvidas constituem-se em uma das formas de assegurar a manutenção da competência da equipe de enfermagem em relação à assistência de enfermagem ${ }^{8}$.

Em geral, na literatura estrangeira, verifica-se ênfase na educação continuada do enfermeiro. Entretanto, no nosso meio, é preciso focalizar todas as categorias de enfermagem. Isto também é verdadeiro para aqueles que atuam nas UTIs, visto que, tanto o enfermeiro, como o técnico e o auxiliar de enfermagem prestam cuidados a pacientes internados nestas Unidades e todos necessitam estar treinados e atualizados. Claramente, há deficiência de literatura nacional a respeito.

Por outro lado, autores estrangeiros estudiosos deste tema, também são unânimes em afirmar que há deficiências mesmo restringindo-se às necessidades do enfermeiro. Assim, não é incomum afirmações como as de WICHOWISKI \& $\mathrm{KUBSCH}^{14}$ sobre a necessidade de educacão continuada na UTI, dada a presença da alta tecnologia e o constante desafio para o enfermeiro, de aprendê-la prontamente. Daí, proporcionar programas de educação continuada que atendam adequadamente às necessidades do enfermeiro, bem como o uso eficiente de tecnologia avançada, particularmente nas UTIs, tem se tornado um desafio tanto para os enfermeiros destas Unidades como para os enfermeiros de educação em serviço.

\footnotetext{
* Parte do Projeto Integrado "Recursos assistenciais em UTI-implicações na qualidade da assistência", financiado pelo CNPq (Processo no. 520.768/94-9)

** Professor Titular da Escola de Enfermagem da USP

*** Professor Doutor do Depto. de Enfermagem Médico-Cirúrgica da Escola de Enfermagem da USP

**** Aluna do Curso de Graduação em Enfermagem da Escola de Enfermagem da USP. Bolsista de Iniciação Científica (CNPq)
} 
No Brasil, com a criação de sociedades de especialistas em enfermagem juntamente com o impulso que elas vem ganhando, principalmente nesta década, torna-se necessário refletir sobre certos aspectos educacionais aí envolvidos. Como preparar os enfermeiros para obtenção do título de especialista em terapia intensiva? Como propiciar a manutenção de enfermeiros de UTI motivados quanto ao seu desenvolvimento profissional e atualização?

Para compreender porque os enfermeiros participam de programas de educação continuada, WADDELL $^{13}$ fez uma meta-análise de 22 pesquisas que abordam este tema. A orientação motivacional foi o fator mais importante na decisão de participar de educação continuada enquanto que outros fatores como os demográficos, incluindo a situação de vida e oportunidades educacionais, apareceram como fortes influenciadores na decisão de participar ${ }^{13}$. Como conciliar todos esses fatores e manter a motivação?

Estudar como os enfermeiros reagem e como resolvem as incertezas advindas da tecnologia não familiar, utilizando a teoria fundamentada nos dados, foi o objetivo de WICHOWSKI \& $\mathrm{KUBSCH}^{14}$. Estas autoras concluíram que a educação continuada planejada é vista como o meio mais efetivo para apreender a tecnologia não familiar; que a educação em serviço no hospital é o meio mais utilizado pelas enfermeiras hospitalares; que a educação informal é também um meio usado para se inteirar das mudanças e tendências tecnológicas; que o material publicado e as informações dos colegas são usados para tomar conhecimento dos avanços. No geral, o conhecimento atualizado acrescido da especialização clínica foram considerados os métodos mais efetivos para controlar a incerteza em um meio onde as mudanças são constantes, como é o da UTI. Como estão as atividades de educação continuada, no nosso meio, seja intra-muros ou extra-muros?

O estudo desenvolvido por GOMES et al. ${ }^{5}$ em 1989, mostrou que havia precariedade nos programas de educação continuada desenvolvidos intra-muro hospitalar. Das 29 UTIs selecionadas arbitrariamente, no âmbito do Município de São Paulo, constatou-se que menos da metade delas, ou seja, 46,4\% mantinham programas de treinamento inicial e somente $14,2 \%$, propiciavam atividades de reciclagem.

Considerando o estágio atual de desenvolvimento da assistência de enfermagem em UTI, julgou-se oportuno analisar também estes aspectos, procurando-se, agora, verificar quais são e quando são desenvolvidos os programas de educação continuada intra-muros para a equipe de enfermagem, bem como, quem os desenvolve. Para complementar, investigou-se ainda o que o enfermeiro tem feito enquanto atividades de educação continuada formal extra-muros.

\section{METODOLOGIA}

\subsection{População e amostra}

A partir de uma lista de hospitais do Município de São Paulo (MSP), fornecida pelo Centro de Informações de Saúde (CIS), da Secretaria de Estado da Saúde de São Paulo e utilizando a técnica de amostragem casual estratificada, foram selecionados $40 \%$ de hospitais com UTI de cada uma das 8 regiões integrantes do SUS deste Município. Como foram identificados 88 hospitais com UTIs, o tamanho da amostra foi de 39 hospitais. Destes, 7 foram excluídos por diversas razões, totalizando no final, 32 hospitais onde foram identificadas 47 UTIs.

\subsection{Métodos}

Os dados foram obtidos por meio de um questionário (Anexo) enviado a cada uma das UTIs amostradas, o qual compunha-se das seguintes partes:

A. Caracterização do hospital e da(s) UTI(s);

B. Composição da equipe de enfermagem;

C. Caracterização dos enfermeiros;

D. Treinamento e reciclagem da equipe de enfermagem.

A coleta de dados foi feita no período de dezembro de 1994 a maio de 1995, tendo sido solicitado ao enfermeiro responsável pela UTI que preenchesse o instrumento. Para otimizar o retorno, o questionário foi entregue em mãos e a sua devolução combinada quanto ao local e data de recolhimento.

\section{RESULTADOS E COMENTÁRIOS}

Do total de 47 UTIs, 43 responderam aos questionários (91,5\%). Como já descrito em estudo anterior $^{7}$ as UTIs pesquisadas localizavam-se principalmente em hospitais particulares $(79,2 \%)$. O número de UTIs variou de 1 a 4 nos diferentes hospitais, sendo mais freqüentes aqueles com uma única unidade $(68,8 \%)$. O total de leitos ativados (424) foi praticamente igual à capacidade instalada (460). Predominaram a UTIs gerais $(62,8 \%)$, as destinadas a pacientes adultos $(51,2 \%)$ $\mathrm{e}$ as que atendiam tanto a pacientes clínicos como cirúrgicos $(95,3 \%)$.

Em relação a treinamento inicial para atuar na UTI, $34(79,1 \%)$ responderam ter um programa específico mas, uma parcela considerável, ou seja, 9 (20,9\%) não o têm. Este é um primeiro aspecto que chama a atenção. Embora não ter um programa específico não seja sinônimo de não ter um treinamento inicial, é difícil imaginar como seria um treinamento inicial informal ou desestruturado. Contudo, comparado ao percentual obtido em estudo de $1989^{5}$ que foi de $46,4 \%$, houve uma melhora considerável. 
Tabela 1 - Duração do treinamento inicial específico segundo categorias da equipe de enfermagem. MSP, 1994/95

\begin{tabular}{ccccccc}
\hline \multirow{2}{*}{ Duração } & \multicolumn{3}{c}{ Categorias } \\
& $\mathrm{N}$ & $\mathrm{E}$ & \multicolumn{2}{c}{ TE/AE } & \multicolumn{3}{c}{ AT } \\
& & $\%$ & $\mathrm{~N}$ & $\%$ & $\mathrm{~N}$ & $\%$ \\
\hline$<1$ mês & 9 & 20,9 & 13 & 30,2 & 5 & 11,6 \\
1 mês & 5 & 11,6 & 6 & 14,0 & - & - \\
2 meses & 1 & 2,3 & 1 & 2,3 & - & - \\
3 meses & 7 & 16,3 & 8 & 18,6 & 4 & 9,3 \\
Conforme necessidade & 5 & 11,6 & 5 & 11,6 & 3 & 7,0 \\
Não se aplica & 14 & 32,6 & 8 & 18,6 & 29 & 67,4 \\
Nulo/embranco & 2 & 4,7 & 2 & 4,7 & 2 & 4,7 \\
\hline TOTAL & 43 & 100,0 & 43 & 100,0 & 43 & 100,0 \\
\hline
\end{tabular}

Ao analisar a presença de treinamento inicial e sua duração por categorias de enfermagem, alguns fatos interessantes se destacam. As categorias com maior cobertura foram as dos técnicos e auxiliares de enfermagem $(76,7 \%)$, sendo que a duração mais freqüente foi a de até um mês $(44,2 \%)$. Na categoria enfermeiro, a cobertura atingida foi $62,7 \%$ e a duração mais freqüente foi igualmente a de até um mês $(32,5 \%)$. Na categoria atendente de enfermagem predominou o não treinamento, isto porque a maioria das UTIs não conta com esta categoria no seu quadro de pessoal.

Comparando estes resultados com os obtidos no estudo anterior ${ }^{5}$ no que se refere aos enfermeiros e duração de seu treinamento, não se observou grandes modificações, já que o treinamento de até um mês foi o preponderante.

Basicamente existem três formas de desenvolvimento das atividades de educação continuada, a saber:

- sistema centralizado, em que as atividades são desenvolvidas pelo enfermeiro de educação continuada; - sistema descentralizado, o qual é realizado pela enfermeira da própria unidade;

- sistema misto, o qual é desenvolvido parcialmente pela equipe da educação continuada e pelas enfermeiras das unidades ${ }^{2}$.

Tabela 2 - Sistemas de desenvolvimento das atividades de educação continuada. MSP, 1994/95

\begin{tabular}{lcc}
\hline \multicolumn{1}{r}{ Sistema } & N & $\%$ \\
\hline - Descentralizado & 23 & 53,5 \\
- Centralizado & 6 & 14,0 \\
- Misto & 5 & 11,6 \\
- Não se aplica & 9 & 20,9 \\
\hline TOTAL & 43 & 100,0 \\
\hline
\end{tabular}

Como pode ser visto na Tabela 2, neste estudo houve predomínio do sistema descentralizado. De acordo com CAVALHEIRO \& COLI $^{2}$, em qualquer um dos sistemas, a participação das enfermeiras das unidades é essencial, tendo em vista o seu contato permanente com os membros da equipe e por ter as melhores condições de perceber a realidade e avaliar as necessidades por eles sentidas.

Além disso, ao participar dessas atividades, elas contribuem para a adequação do conteúdo programático, uma vez que conhecem todas as atividades a serem desenvolvidas.

Tabela 3 - Programas de atualização e freqüência de oferecimento. MSP, 1994/95

\begin{tabular}{lcc}
\hline \multicolumn{1}{r}{ Atualização } & N & $\%$ \\
\hline Sim, periódica & 10 & 23,3 \\
Sim, ocasional & 8 & 18,6 \\
Não & 25 & 58,1 \\
\hline TOTAL & 43 & 100,0 \\
\hline
\end{tabular}

Em relação aos programas de atualização, como observado no treinamento inicial, houve incremento de $14,2 \%$ do estudo anterior ${ }^{5}$ para o presente, que foi de $41,9 \%$. Contudo, verificando-se que $58,1 \%$ das UTIs não ofereciam programas de atualização, pode-se considerar que o percentual de $41,9 \%$ é uma proporção ainda baixa, tendo em vista a necessidade de atualização constante para a atuação em UTI.

No estudo desenvolvido por SULLIVAN \& $\mathrm{BREU}^{12}$ em 1982 para conhecer como a enfermagem era praticada nas UTIs norte-americanas, estas autoras constataram que $83 \%$ dos hospitais proporcionavam orientação para a equipe de enfermagem, sendo que havia 
uma correlação entre o fornecimento de orientação e o tamanho do hospital. Em geral, os hospitais maiores deliberavam mais orientação que os menores, assim como a educação em serviço era mais freqüente nos hospitais maiores que nos menores. Havia também diferenças de ordem regional.

Um outro aspecto também detectado naquele estudo foi que cerca de $2 / 3$ dos hospitais enviavam seus enfermeiros para outras instituições para atividades de educação continuada e que isto era menos freqüente nos hospitais com mais de 400 leitos. Dos hospitais estudados, somente $23 \%$ treinavam enfermeiros de outros hospitais e esta atividade guardava relação com o tamanho do hospital. Quanto maior o hospital, maior era a oferta deste tipo de serviço.

No presente estudo, das $18(41,9 \%)$ UTIs que promoviam programas de atualização, tal como no treinamento inicial, o sistema predominante foi o descentralizado, ou seja, aquele desenvolvido pelas enfermeiras da própria Unidade. Quanto ao conteúdo dos programas de atualização, das 18 UTIs que ofereciam esta programação, 3 não especificaram seu conteúdo. As demais, em sua grande maioria, relataram a revisão de técnicas e rotinas e a atualização em patologias. Somente uma Unidade informou ter conteúdo visando melhoria de atendimento e qualidade do cuidado.

Observa-se, assim, uma ênfase em conteúdos voltados para os aspectos clínicos e administrativos da assistência intensiva, os quais certamente atendem às características do tratamento intensivo. Entretanto, considera-se também como de importância relevante a instrumentalização da equipe de enfermagem quanto a outros aspectos da assistência ao paciente crítico, como os psicossociais, emocionais e ético-legais, tão presentes no cotidiano das UTIs.

Tabela 4 - Avaliação periódica de desempenho da equipe de enfermagem. MSP, 1994/95

\begin{tabular}{lcc}
\hline \multicolumn{1}{c}{ Periodo } & $\mathrm{N}$ & $\%$ \\
\hline - Probatório & 3 & 7,0 \\
- Entre 3 e 6 meses & 3 & 7,0 \\
- A cada 6 meses & 5 & 11,6 \\
- Entre 6 e 12 meses & 8 & 18,6 \\
- A cada 12 meses ou & 8 & 18,6 \\
mais & 4 & 9,3 \\
- Não definido & 12 & 27,9 \\
- Nunca & & \\
\hline TOTAL & 43 & 100,0 \\
\hline
\end{tabular}

Como mostram os dados da Tabela 4, há variação quanto à periodicidade das avaliações sendo mais freqüente entre 6 e 12 meses e a cada 12 meses ou mais.
É interessante observar também que cerca de $28 \%$ das UTIs nunca realizam avaliações de desempenho.

Verificou-se ainda que, onde a avaliação periódica é realizada, ou seja, em cerca de $2 / 3$ das unidades, ela é feita basicamente pelo enfermeiro da UTI ou pelo enfermeiro-chefe.

Considerando os conteúdos dos programas de atualização detectados, aliada à periodicidade das avaliações tal como vem ocorrendo, acredita-se que é preciso refletir sobre o significado do que sejam necessidades de aprendizado e consequentemente, de avaliação.

KRISTJANSON \& SCANLAN ${ }^{8}$ desenvolveram um estudo para identificar aspectos metodológicos e conceituais e as instrumentações importantes para conduzir uma avaliação das necessidades de educação continuada dos enfermeiros. Baseadas na revisão de literatura elas agruparam quatro importantes temas a serem investigados: a importância de clarificar e definir o constructo "necessidade" ao conduzir a avaliação de necessidades; as variáveis que influenciam a participação nos programas de educação continuada; os métodos para avaliar necessidades e o "marketing" desses programas.

$\mathrm{Na}$ mesma linha de raciocínio, para Atwood \& Elis, citadas por KRISTJANSON \& SCANLAN ${ }^{8}$, há quatro tipos de necessidades:

- as reais, que são as deficiências atuais presentes que, podem ou não ser reconhecidas por aqueles que apresentam essas necessidades;

- as educacionais, que são resultados de deficiências educacionais anteriores e podem ser solucionadas com o aprendizado;

- as educacionais reais, que se referem à compreensão, habilidades ou atitudes específicas que estão faltando e podem ser satisfeitas com a aprendizagem;

- as sentidas, que são as pessoais.

Estas categorias podem ser indistintas e difíceis de serem diagnosticadas. A questão crucial envolve identificar tanto as necessidades sentidas como as normativas institucionais. Assim é importante que haja um diálogo aberto entre aquele que aprende e aquele que programa as atividades de educação continuada, para que se possa atingir um objetivo comum.

Tais afirmações mostram as dificuldades inerentes a uma avaliação adequada e a complexidade das necessidades a serem identificadas. Reforçando a problemática da avaliação, na revisão de literatura foi possível detectar a ênfase dada na orientação e desenvolvimento do enfermeiro que se inicia no cuidado intensivo. Entretanto, segundo FEENEY \& BENSONLANDAU $^{3}$ pouca atenção tem sido dada ao acompanhamento e avaliação da competência do enfermeiro de UTI, após o período de treinamento inicial. 
Para essas mesmas autoras ${ }^{3}$, os enfermeiros administradores devem assumir a responsabilidade de assegurar que o enfermeiro de UTI tenha os conhecimentos e as habilidades necessários para cuidar do paciente criticamente enfermo. Eles devem implementar a avaliação fundamentada na competência e programas que permitam validar a competência da equipe de enfermagem, bem como, recomendar programas educacionais nas áreas detectadas como deficitárias.

Igualmente, para HOUGE \& DEINES $^{6}$ o estabelecimento da competência clínica é muito importante tanto para as enfermeiras que atuam nas unidades de cuidados críticos, como para as instituições empregadoras e para os consumidores dos serviços de saúde. A avaliação cuidadosa, seja do enfermeiro iniciante como do experiente, deve ser desenvolvida de forma não ameaçadora, a fim de que as habilidades cognitivas e psicomotoras possam ser objetivamente avaliadas. Afinal, o objetivo maior deve ser sempre visto em termos de promoção de uma alta qualidade de cuidado ao paciente.

Como já mencionado anteriormente, o outro objetivo deste estudo foi o de identificar a situação da educação continuada formal extra-muros na categoria enfermeiro.

No presente estudo foram identificados 251 $(18,9 \%)$ enfermeiros dentre o total de 1328 elementos da equipe de enfermagem. A maior proporção foi representada pelos auxiliares de enfermagem, com 912 $(68,7 \%)$ profissionais. Os técnicos de enfermagem somaram $54(4,1 \%)$ e os atendentes, $111(8,3 \%)$.

Dos 251 enfermeiros lotados nas UTIs estudadas, 237 responderam os quesitos quanto às suas características, as quais podem ser vistas na Tabela 5 .

Tabela 5 - Distribuição dos enfermeiros segundo faixa etária e tempo de formado. MSP, 1994/95

\begin{tabular}{|c|c|c|c|c|c|c|}
\hline \multirow[b]{2}{*}{ Faixa etária } & \multicolumn{4}{|c|}{ Tempo de formado } & \multirow[b]{2}{*}{ Em branco } & \multirow[b]{2}{*}{ TOTAL } \\
\hline & $<1$ & $1|--| 5$ & $6|--| 10$ & 11 ou + & & \\
\hline $20 \mid--25$ & 4 & 20 & - & - & - & 24 \\
\hline $26 \mid--30$ & 1 & 51 & 30 & - & 1 & 83 \\
\hline $31|--| 35$ & - & 12 & 37 & 8 & 1 & 58 \\
\hline $36 \mid--40$ & 1 & 9 & 6 & 15 & - & 31 \\
\hline 41 ou + & - & 5 & 4 & 10 & 3 & 22 \\
\hline Em branco & 0 & 3 & 1 & 1 & 14 & 19 \\
\hline TOTAL & 6 & 100 & 78 & 34 & 19 & 237 \\
\hline
\end{tabular}

Por esta tabela é possível verificar que a grande maioria localiza-se na faixa etária de até 35 anos e que tem entre 1 e 10 anos de formado. Poder-se-ia dizer que trata-se de uma população que se encontra na fase ascendente de sua trajetória profissional e consequentemente, bastante receptiva a programas de atualização e de desenvolvimento profissional.

Ao investigar que tipos de educação continuada formal extra-muros são por eles procurados, constatou- se predomínio nos cursos de pós-graduação senso lato.

Dos 237 enfermeiros, 119 (50,2\%) assinalaram ter feito cursos de pós-graduação senso lato, ou seja, especialização ou aprimoramento em enfermagem médico-cirúrgica (MC) ou em outra área, ou tanto em enfermagem médico-cirúrgica como em outra área. Somente 2 assinalaram ter feito pós-graduação senso estrito, sendo um só mestrado e o outro, mestrado e especialização em enfermagem médico-cirúrgica.

Tabela 6 - Distribuição dos enfermeiros $(n=119)$ segundo cursos de pós graduação senso lato e tempo de formado. MSP, 1994/95

\begin{tabular}{|c|c|c|c|c|c|c|}
\hline \multicolumn{7}{|c|}{ Tempo de formado } \\
\hline \multirow[t]{2}{*}{ Cursos } & \multirow{2}{*}{$1|--| 5$} & \multirow[t]{2}{*}{$6|--| 10$} & \multirow[t]{2}{*}{11 out +} & \multirow{2}{*}{ Em branco } & \multicolumn{2}{|c|}{ TOTAL } \\
\hline & & & & & $\mathrm{N}$ & $\%$ \\
\hline Esp./Apr.MC & 24 & 15 & 7 & - & 46 & 38,7 \\
\hline Esp./Aproutras áreas & 10 & 20 & 11 & 2 & 43 & 36,1 \\
\hline Esp./Apr.MC e outras áreas & 4 & 5 & 6 & - & 15 & 12,6 \\
\hline Esp./Apr em áreas não identificadas & 7 & 6 & 2 & - & 15 & 12,6 \\
\hline TOTAL & $45(37,8)$ & $46(38,7)$ & $26(21,8)$ & $2(1,7)$ & 119 & 100,0 \\
\hline
\end{tabular}


Conforme mostram os dados da Tabela 6, dos enfermeiros que assinalaram ter cursos de especialização ou aprimoramento, $61(46+15)$ os fizeram só em enfermagem médico-cirúrgica ou nesta e também em outra área. Considerada a totalidade de enfermeiros deste estudo $(n=237)$ e que eles atuam em unidades altamente especializadas, verifica-se que a proporção ainda é baixa.

Um aspecto não investigado foi o relativo aos métodos de atualização utilizados pelo enfermeiro de UTI. Na perspectiva de que a educação continuada possa se tornar requisito para titulação ou para sua renovação, buscou-se na literatura as possíveis influências da atualização mandatória, bem como, a associação desta com a rotatividade ou não de plantões e quem arca com os custos das atividades de educação continuada.

Tendo a educação continuada se tornado mandatória para re-licenciamento em um dos estados americanos, CANNON \& WATERS ${ }^{1}$ investigaram por meio de uma amostra de 535 enfermeiros daquela localidade, como eles responderiam a este requisito. Os resultados obtidos mostraram que, enquanto $40 \%$ dos enfermeiros indicaram que poderiam aumentar sua participação nas atividades de educação continuada, mais da metade da amostra indicou que a participação continuaria a mesma. O método preferencial indicado foi a conferência, seguida do uso de video-tape ou material impresso. $\mathrm{O}$ menos escolhido foi o audio-tape. A área mais selecionada foi a prática clínica, seguida da administração em enfermagem.

Com relação à educação continuada mandatória como requisito para re-licenciamento do enfermeiro para o exercício profissional, GESSNER \& ARMSTRONG ${ }^{4}$, estudaram as atividades de leitura dos enfermeiros $(\mathrm{N}=269)$ em 6 estados americanos com este tipo de legislação e o mesmo número de enfermeiros em estados sem este requisito. Os resultados obtidos mostraram que os dois grupos usam duas vezes mais tempo lendo literatura geral do que profissional. Considerando a escolha do método para atividades de educação continuada, as autoras verificaram que ambos preferem a conferência. Ler foi a segunda escolha para aqueles de estados com legislação mandatória e terceira, nos estados onde a educação continuada é voluntária. Todavia, nos estados onde a educação continuada era voluntária, os enfermeiros despendiam tempo um pouco maior para leitura profissional.

Uma questão que é sempre lembrada é a influência da rotatividade dos plantões, comum entre enfermeiros que trabalham em UTIs e que poderia atuar como fator para a baixa freqüência em atividades de educação continuada.

STARING $^{11}$ desenvolveu uma pesquisa em dois hospitais universitários onde os enfermeiros trabalhavam em turnos fixos, ou seja, diurno ou noturno e em tempo integral. A amostra contou com 159 enfermeiros destas UTIs. Como instrumento de medida, ela utilizou a Escala de Participação Educacional que mede fatores motivacionais e é classificada em 6 dimensões: contato social, estimulação social, desenvolvimento profissional, serviço comunitário, expectativas externas e interesse cognitivo. Os resultados mostraram que os enfermeiros eram primariamente motivados por interesse cognitivo e desenvolvimento profissional. Aprender mais para ter mais segurança foi o fator mais freqüente e a forma de obtenção, os cursos relacionados com conhecimentos e habilidades. Nenhuma diferença estatística foi constatada entre enfermeiros do plantão diurno e do noturno. A autora comenta que isto deve ter ocorrido porque o plantão era fixo.

E se o plantão for rotativo? De uma certa forma esta é uma prática bastante comum no nosso meio. Partindo do pressuposto de que a educação continuada é um direito individual e uma obrigação do profissional em exercício, SCHOEN \& MORGAN ${ }^{10}$ investigaram quem paga os custos da educação continuada já que elas não encontraram dados na literatura que respondessem a esta questão. O estudo foi desenvolvido contando com uma população de 1171 enfermeiros que haviam realizado alguma atividade de educação continuada entre 1988 a 1990, em um estado onde esta atividade não é mandatória.

Os resultados obtidos mostraram que quase metade dos enfermeiros tiveram as atividades de educação continuada paga pelo empregador. As áreas mais privilegiadas foram as ambulatoriais, de saúde pública e saúde da comunidade e de cuidado domiciliar e a menos privilegiada foi a das instituições de ensino. Enfermeiros de UTI e de reabilitação receberam menos suporte e os administradores, mais. Destes, o enfermeiro administrador foi o que recebeu mais suporte, seguido do enfermeirochefe e depois, o enfermeiro assistencial.

Tais resultados mostraram que o apoio dos empregadores esteve correlacionado com a natureza do emprego, a qual foi fortemente influenciada pelo cargo ou função e salário do enfermeiro. Foram ainda consistentes de que o apoio do empregador baseia-se mais no cargo ou função do profissional do que nas reais necessidades do enfermeiro.

De uma forma geral, dificuldades existem e continuarão a existir na implementação de programas efetivos de educação continuada. Nesta perspectiva, uma forma de conciliar necessidades sentidas e minimizar deficiências foi estudada por MALONEY \& KANE ${ }^{9}$. Partindo da premissa de que as necessidades sentidas produzem maior motivação para aprender e do dilema de ter um instrumento capaz de identificar as reais deficiências de conhecimento e encontrar as necessidades sentidas, estas autoras desenvolveram um questionário com questões diretas cobrindo aspectos clínicos e questões 
profissionais no cuidado de neonatos em uma UTI neonatal. Os respondentes, enfermeiros desta UTI, assinalaram sua capacidade de responder totalmente, parcialmente ou serem incapazes de responder a cada uma das questões. Com isto, a comissão de educação continuada pode identificar as falhas de conhecimento na prática assistencial diária desta equipe de enfermagem e pode implementar um programa mais adequado às reais necessidades daquela clientela. Corroborando isto, os enfermeiros indicaram alto nível de satisfação. Segundo estas mesmas autoras, este sistema tem sido utilizado a cada dois anos e tem mostrado que atinge melhor as necessidades da equipe de enfermagem

São, pois, grandes os desafios da educação continuada, entre outras, conciliar as necessidades sentidas pelos enfermeiros com as normativas institucionais, manter formas de avaliação que visem promoção e desenvolvimento, favorecer condições materiais e de tempo para cumprimento desta que é um direito individual e ao mesmo tempo, uma responsabilidade profissional.

\section{CONCLUSÕES}

- Do total de UTIs estudadas, 34 (79,1\%) responderam ter treinamento inicial específico para a equipe de enfermagem. As categorias com maior cobertura foram as do técnico de enfermagem e do auxiliar de enfermagem com $76,7 \%$ e a duração mais freqüente foi a de até um mês $(44,2 \%)$.

- Os programas de atualização são desenvolvidos por 18 $(41,9 \%)$ das UTIs e estes enfocam principalmente, revisão de técnicas e rotinas e atualização em patologias.

- Tanto o treinamento inicial como os programas de atualização são desenvolvidos predominantemente pelo enfermeiro de UTI.

- Em relação ao enfermeiro, 50,2\% assinalaram ter cursos de especialização ou aprimoramento em enfermagem médico-cirúrgica ou em outra área, ou tanto em enfermagem médico-cirúrgica como em outra área.

\section{CONTINUING EDUCATION OF ICU NURSING STAFF IN SÃO PAULO CITY}

This study is part of a project about ICU's characteristics in São Paulo city. This article describes the continuing education programs for nursing staff. 43 ICUs were analysed and the questionnaire answered by the ICU nurse cordinator was used to collect data. Results showed that $34(79,1 \%)$ of the ICUs have initial program for training each nursing staff category and 18 (41,9\%) had regular continuing education program focusing primarily on nursing procedures and routines and the update in pathologies. Continuing education programs are developed primarily by ICU's nurses. 50,2\% of the nurses answered that they attended specialisation/ extension courses too, in Medical Surgical Nursing or in other areas or in both areas. Due to this result and the development of the ICU's nurses as specialists, some suggestions are presented to improve the continuing education program in these Units.

KEY WORDS: continuing education, nursing staff, intensive care units

\section{EDUCACIÓN CONTINUA DEL EQUIPO DE ENFERMERÍA EN UNIDADES DE TRATAMIENTO INTENSIVO DEL MUNICIPIO DE SÃO PAULO}

Este estudio hace parte de un proyecto sobre caracterización de las UTIs en el Municipio de São Paulo, en lo que se refiere a la estructura fisica y los recursos humanos y materiales. Los aspectos relativos a las actividades de educación continua del equipo de enfermeria son analizados en este estudio. La muestra estudiada fue de 43 UTIs y un cuestionario respondido por la enfermera responsable por la Unidad, el instrumento usado para la recolección de los datos. En 34 (79,1\%) de las UTIs estudiadas se realizaban entrenamiento inicial especifico y en 18 (41,9\%), programas de atualizacción, los cuales enfocaban, principalmente, la revisión de técnicas y rutinas y la atualizacción de patologias. Las actividades de educación continua son desarrolladas, predominantemente, por los enfermeros de las UTIs. 50,2\% de los enfermeros respondieran haber frecuentado cursos de especialización o aprimoramiento en Enfermería Médico-Quirúrgica o en otras áreas. Frente a lo detectado y considerando el desarrollo del enfermero de UTI como el de un especialista, se presentan algunas sugestiones para el mejoramiento de las actividades de la educación continua en estas Unidades.

TÉRMINOS CLAVES: educación continua, equipo de enfermería, unidades de tratamiento intensivo 


\section{RECURSOS ESTRUTURAIS DAS UTI(s) DO MUNICÍPIO DE SÃO PAULO}

\section{A. Caracterização do Hospital e da(s) UTI(s)}

\section{A.1 - Identificação da Instituição}

- Nome:

- Tipo de hospital:

( ) público

( ) privado

- Número de leitos do hospital: capacidade total:

capacidade ativada:

- Atendimento: ( ) geral

( ) especializado. Especifique:

- $\mathrm{N}^{\mathrm{o}}$ de UTIs:

\section{A.2 - Caracterização individual da UTI}

- Atendimento: ( ) geral

( ) especializado. Especifique:

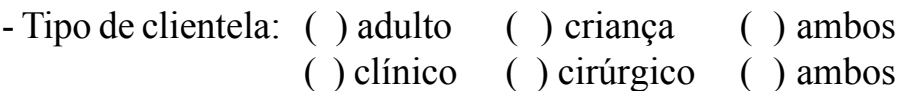

- Número de leitos: Total:

Ativados

B. Composição da equipe de enfermagem

\begin{tabular}{|c|c|c|c|c|c|}
\hline $\begin{array}{c}\text { Turno } \\
\text { Dotação } \\
\text { Componentes }\end{array}$ & $\operatorname{Sim}_{\left(n^{o}\right)^{M} \text { Não }}$ & $\begin{array}{c}\mathrm{T} \\
\operatorname{Sim}\left(n^{0}\right) \quad \text { Não }\end{array}$ & $\begin{array}{c}\text { Npar } \\
\operatorname{Sim}\left(\mathrm{n}^{\mathrm{o}}\right) \quad \text { Não }\end{array}$ & $\begin{array}{l}\text { Nímpar } \\
\operatorname{Sim}\left(n^{\circ}\right) \quad \text { Não }\end{array}$ & $\begin{array}{l}\text { Quadro } \\
\text { Atual }\end{array}$ \\
\hline Enf.Chefe & & & & & \\
\hline Enf.Assistencial & & & & & \\
\hline Téc.Enfermagem & & & & & \\
\hline Aux.Enfermagem & & & & & \\
\hline Atend.Enfermagem & & & & & \\
\hline
\end{tabular}

\section{Caracterização dos enfermeiros}

\begin{tabular}{|c|c|c|c|c|c|c|c|c|}
\hline \multirow[t]{2}{*}{ Enfermagem } & \multirow[t]{2}{*}{ Idade } & \multirow{2}{*}{$\begin{array}{l}\text { Tempo de } \\
\text { formado }\end{array}$} & \multirow{2}{*}{$\begin{array}{l}\text { Tempo de } \\
\text { trabalho em } \\
\text { UTI }\end{array}$} & \multicolumn{5}{|c|}{ FORMAÇÃO } \\
\hline & & & & Mestrado & Doutorado & Especialização & Aprimoramento & Outros \\
\hline \multicolumn{9}{|l|}{1} \\
\hline \multicolumn{9}{|l|}{2} \\
\hline \multicolumn{9}{|l|}{3} \\
\hline \multirow{2}{*}{\multicolumn{9}{|c|}{$\frac{4}{5}$}} \\
\hline & & & & & & & & \\
\hline \multicolumn{9}{|l|}{6} \\
\hline 7 & & & & & & & & \\
\hline 8 & & & & & & & & \\
\hline
\end{tabular}




\section{Treinamento e reciclagem da equipe de enfermagem da UTI}

- Há um treinamento específico para assumir as funções na UTI?

( ) Não

( ) $\mathrm{Sim}$

Para que categoria?

Quem o realiza?

- Qual a duração desse treinamento?

- Enfermeiros

- Técnico/auxiliar

- Atendente

- Existe avaliação periódica de desempenho?

( ) Não

( ) Sim Com que freqüência?

Quem o realiza?

- Existe um programa de reciclagem específico para o pessoal de enfermagem em UTI?

( ) Não

( ) $\operatorname{Sim}$

No que se constitui?

Com que freqüência?

Quem o realiza?

\section{REFERÊNCIAS BIBLIOGRÁFICAS}

01. CANNON, C. A.; WALTERS, L.D. Preparing for mandatory continuing education - assessing interests. J. Contin. Educ. Nurs., v. 24, n. 4, p.148-152, 1993.

02.CAVALHEIRO, E.R.A.; COLI, R.C.P. Treinamento em enfermagem. Rev. Paul. Enfermagem, v. 12, n. 2, p. 57-59, 1993.

03. FEENEY, J.; BENSON- LANDAU, M. Competencybased evaluation: not just for new nurses. Dimens. Crit. Care Nurs. , v. 6, n. 6, p. 368375, 1987.

04.GESSNER, B.A.; ARMSTRONG, M.L. Reading activities of staff nurses from states with mandatory or voluntary continuing education. $\mathbf{J}$. Contin. Educ. Nurs., v.23, n. 2, p.76-80, 1992.

05.GOMES, A.M. et al. Tendências no treinamento e reciclagem do pessoal de UTI. /Trabalho apresentado no III Encontro de Enfermagem em terapia Intensiva, São Paulo, 22 a 24 de outubro de $1987 /$.

06. HOUGE, M.C. Verifying clinical competence in critical care. Dimens. Crit. Care, v.6, n. 2, p. 102-110, 1987.

07. KIMURA, M.; KOIZUMI, M.S.; MARTINS, L.M.M. Caracterização das Unidades de Terapia Intensiva no município de São Paulo. Panam. J. Trauma, v.5, n.2, p.28, 1995.
08. KRISTJANSON, 1.J.; SCANLAN, J.M. Assessment of continuing nursing education needs: a literature review. J. Contin. Educ. Nurs., v.23, n. 4, p.156160, 1992.

09. MALONEY, P.; KANE, J.W. An innovative solution to assessing staff learning needs. J. Contin. Educ. Nurs., v.26, n.2, p.67-72, 1995.

10. SCHOEN, D.C.; MORGAN, A. P. Who pays for continuing education in nursing. J. Contin. Educ. Nurs., v. 24, n. 1, p. 13-16, 1993.

11. STARING, S. L. Addressing the educational needs of shiftworkers: should shift be a consideration? J. Contin. Educ. Nurs., v. 26, n. 2, p.79-83, 1995.

12. SULLIVAN, S.; BREU, C. Survey of critical care nursing practice. Part IV - staffing and training of intensive care unit personnel. Heart \& Lung, v.11, n. 3, p. 237-241, 1982.

13. WADDELL, D.L. Why do nurses participate in continuing education? a meta-analysis. J. Contin. Educ. Nurs., v. 24, n. 2, p. 52-56, 1993.

14. WICHOWSKI, H.C.; KUBSCH, S. How nurses react to and cope with the uncertainty of unfamiliar technogy: validation for continuing education. $\mathbf{J}$. Contin. Educ. Nurs., v. 26, n. 4, p. 174-178, 1995. 